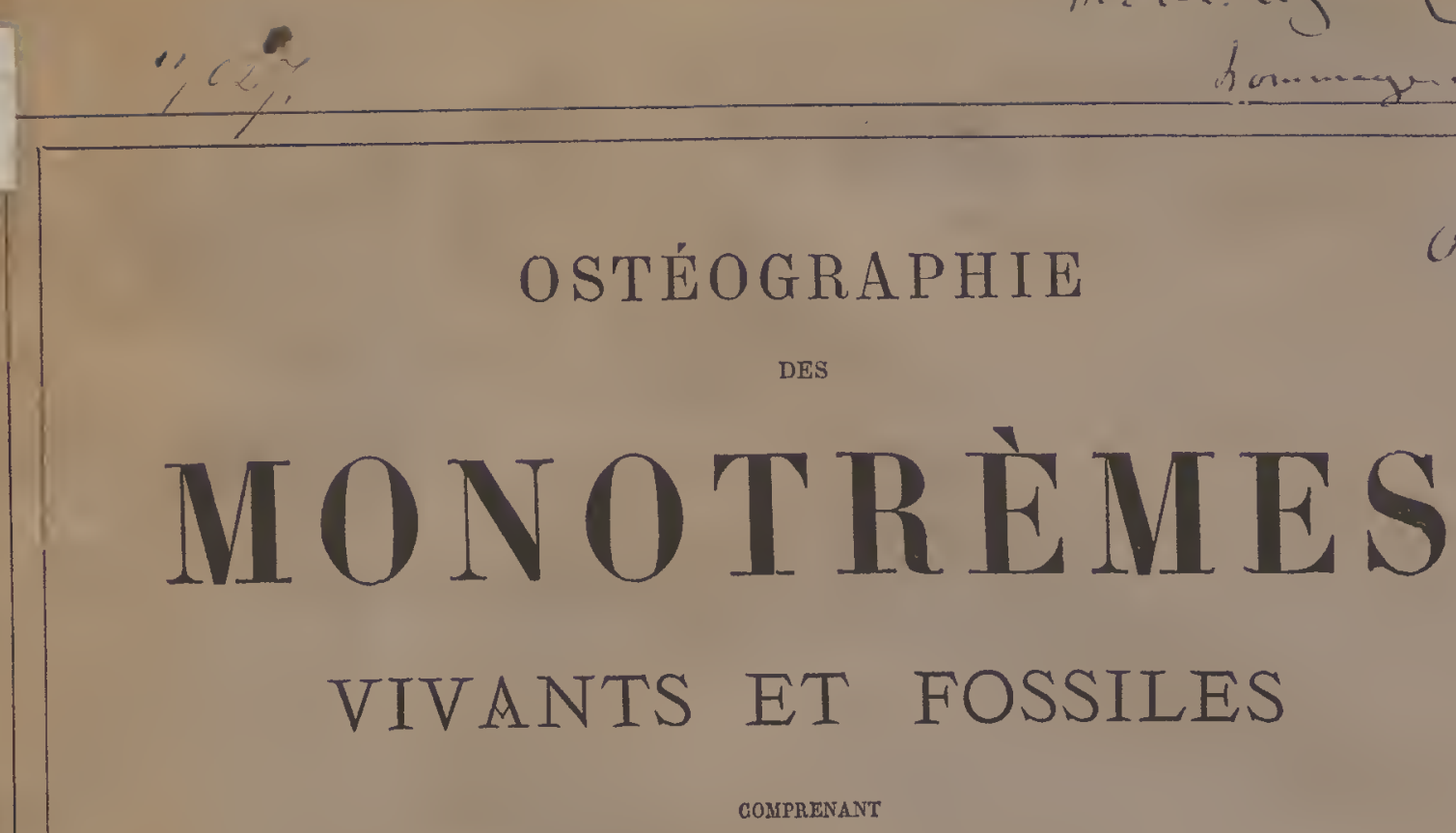

LA DESCRIPTION ET L'ICONOGRAPHIE

D $v$

SQUELETTE ET DU SYSTEME DENTAIRE DE CES ANIMAUX

AINSI QUE DES DOCUMENTS RELATIFS A LEUR HISTOIRE NATURELLE

PA

PAUL GERVAIS

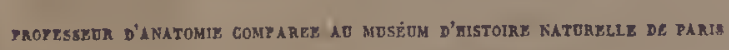
MBMBRE DE L'TNSTITUT (ACADEMIE DES SCTINGES)

\title{
TEXTE
}

PARIS

ARTHUS BER'TRAND, LIBRAIRE-EDITEUR, LIBRAIRIE SCIENTIFIQUE ET MARTTIME Ino Hautefoulle, 21 .

1877-1878

\begin{tabular}{|c|c|}
\hline 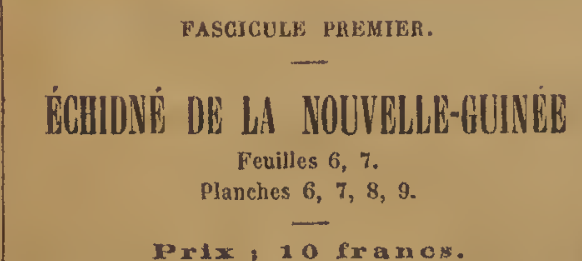 & 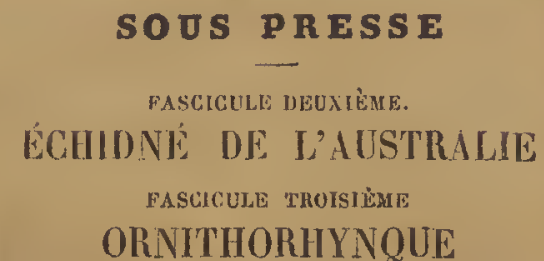 \\
\hline
\end{tabular}




$$
x-G 385 F
$$




\section{CHAPITRE DEUXIEME.}

ARTHUS BERTRAND, ÉDITEUR

IBATRIE MARITIME ET SCIENTIFIQUE

21, rue Inutefeuilke, in Paris.

\section{OSTÉOGRAPHIE}

\section{DES CÉTACÉS VIVANTS ET FOSSILES}

COMPRENANT

\author{
LA DESCRIPTION ET L'ICONOGRAPIIE
}

\section{DU SQUELETTE ET DU SYSTEME DENTAIRE} DE GES ANIMAUX

AINSI QUE DES DOCUMENTS RELATIFS A LEUR HISTOIRE NATURELLE

$$
\text { PAR MM. }
$$

VAN BENEDEN,

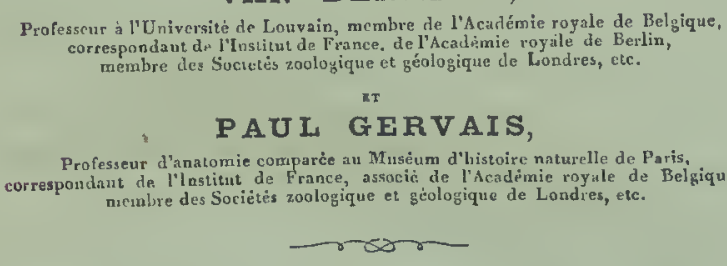

\section{PROSPECTUS.}

Destinés à vivre an sein des mers, les Cétacés ont une certaine ressemblance extéDestines a vissons; mais ils sont bien éloignés d'avoir la structure anatomique de ces animaux. Leurs poumons conformés comme ceux des mammifères, l'élévation de leur température, lour cœur divisé en quatre cavités, le volume et la disposition de de leur température, lcur ceeur intelligence dont ils sont doués, leur mode de reproleur cerveau, l'incontestable ilacentaire : tout, dans leur organisation cornme dans duction à la fois vivipare ct placentaire : tout, dans leur organisation cornme dan leurs fonctions, démontre que ce sont bien des mammifères, et la présence de mamelles, destinécs a élaborer la première nourriture de lcurs peti
des caractères qui les rattachent anx vertébrés de cetle classe.

Les conditions spéciales de l'habitat des Cótacés expliquent assez les parlicularités Lesinguent ces animaux des autres mamınifères et en font un groupe à la fois qui distinguent ces animaux des autres namitude présente-t-clle un intúrêt vẻritable. naturel et facile à reconnaitre; aussi leur étnde presente-t-clle un intere justifierait L'inıportance des produits que plusieurs genres fournissent à l'industric justifierait

\section{E-GUINÉE}

sus) Lawesii.

aotrèmes de la famille ouvelle-Hollande, mais ne publié la description ippartient au Musée de ant aussi eette origine, itannique pour l'avanait à l'espèee, différente mait de signaler la préte seeonde espéce, qui celle dont le Musée de te de l'Échidné d'Auserver deux des Échidnés

lles formes d'Échidnidés nous 1 sujet de l'histoire naturelle l'Ostéographie des „ITonotrèmes de consacrer aux Mammiferes $t$ le troisième dans lequel il eurement, dans le courant de

(30 novembre 1877.)

us (Echidna) proveniente dalla di St. nat. di Genova, t. IX, 
depuis le commencement de notre siècle, puisque la notion de leur structure ou celle de leurs mœurs et de leur répartition dans les différentes mers peut rendre leur capture plus facile et indiquer au commerce de nouvelles sources de richesses.

Mais le naturaliste qui veut entreprendre de semblables recherches ne tarde pas à rencontrer des obstacles sans nombre. A cause de leur énorme taille autant que par la nature des parages qu’ils fréquentent, les Cétacés sont difficilement accessibles à nos moyens d'observation, et il est bien rarement possible de préparer avec tout le soin désirable la dépouille des exemplaires qu'on a réussi a se procurer. Les peaux qu'on en avait d'abord recueillies el conservẻes dans plusieurs musées ne peuvent donner qu'une idée fort incompléte des caractères propres aux différents genres de cet ordre et aux espèces qui composent ces genres. Ajoutons que de semblables collections fussent-elles toujours possibles, peu de musées seraient assez riches pour les entreprendre ou assez vastes pour les loger, de manière $\dot{a}$ en rendre l'examen profitable. Du reste, des difficultés souvent insurmontables s'opposent à ces sortes de prẻparations. Les squelettes eux-mêmes sont coùteux, d'une installation également gênante, et, comme l'importance des indications que l'on peut en tirer a été longtemps méconnue, on s'est jusque dans ces derniers temps assez peu préoccupé de les conserver. Quant aux parties molles, elles ont rarement attiré l'attention des naturalistes, ce qui s'explique par les obstacles plus grands encore qui s'opposent à leur préparation. Aussi la cétologie est-elle restée longtemps stationnaire, ct il y a quelques années seulement la nomenclature des baleines, aussi bien que celle des cachalots, des dauphins ou des genres qui s'en rapprochent, élait encore dans un éta d'imperfection regrettable.

Cependant l'examen ostéologique des Cétacés devait foumir aux savants les moyens de faire accomplir á la science des progrès réels, et c'est à ses indications que nous devons de pouvoir formuler désormais avec cerlitude la diagnose de la plupart des animaux de ce groupe; elle a également permis d'en établir la classification d'une maniére naturelle et de rectifier leur nomenclature. G. Cuvier n'a pas tardé à reconnaitre les avantages que l'on peut en tirer, et Frédéric Cuvier a dit avec raison, en parlant des résultats consignés dans le cinquième volume des Ossements fossiles : "Je crois que le premier exemple bon á suivre a élé donné par mon frère. " Il est également dans le vrai lorsqu'il ajoute: "Ses travaux seront longtemps encore la base de cetle branche importante de la zoologie.

Mais, depuis l'époque où G. Cuvier a ėcrit, les collections se sont enrichies d'un nombre considérable de piéces relatives aux Cètacés alors connus, et des espèces, dans certains cas même des genres entièrement nouveaux ont été découverts. Aussi, quoique toutes les lacunes iaissées par ce zoologiste célébre n'aient pas pu être comblées, on est dés à prẻsent en mesure d'ajouter beaucoup de documents à ceux qu'il a publiés, et d'asseoir sur des bases plus larges qu'il n'avait pu le faire l'histoire des mammiféres marins. Des travaux considérables consacrés à ces animaux ont été publiés, parliculiérement en Danemark et en Angleterre, el ces travaux ont notablement augmenté la somme de nos connaissances. Chaque jour les musées continuent à s'enrichir de piéces nouvelles, et une histoire à peu près complète du groupe entier peut maintenant être tentée avec quelque chance de succès.

M. Eschricht, savant analomiste de Copenhague, dont la science regrette la perte récente, a rẻussi à fournir à la cétologic des documents dont la valeur est réellement exceptionnelle. Les recherches de son compatriote, M. le professeur Reinhardt, et celles de M. Lilljeborg, d'Upsal, méritent aussi unc mention particulière. Il en est 


\section{CHAPITRE DEUX IÈME.}

\section{$-3-$}

de même des importantes publications de MM. J. E. Gray et Flower. Ces savants et plusieurs autres en France, en Belgique, en Allemagne, aux Etats-Unis, ont aussi ajouté beaneoup de faits curieux à ceux dont la cètologie était redevable aux travaux des auteurs antérieurs.

Mieux renseigné que ne l'avaienı été ses prédécesseurs, Lacépède, P. Camper et G. Cuvier, au sujet des mœurs de ees gigantesques animaux, M. Eschricht a pu se faire une idée plus exacle des diffèrentes espèces propres aux régions boréales, et la baleine du Groenland a èté de sa part l'objet de reeherehes approfondies. Les observations de Seoresby, reeueillies dans les parages mêmes oủ se fait la pêche, ont été pour lui une souree d'indications fécondes, et il a eu la bonne fortune d'obtenir de M. Holboll, gouverneur du Groenland, plus de matérianx relatifs à l'anatomie des Cétaeés que n'en avait jusqu'alors réuni aucun zoologiste.

Ces préeieuses eollections se seraient aeerues bien davantage sans la mort de l'aetif et intelligent naturaliste qui les reeueillait aver tant de zèle et tant de dévouement; mais M. Holboll a péri au milieu des banquises en retournant au Groenland, après être venu à Copenhague pour se reposer quelque temps de ses fatigues. Depuis lors la seience a été privée des observations si exaetes qu'il poursuivait ainsi que des objets en nature, squelettes d'ảges el de sexes différents appartenant à toutes les espèces, préparations anatomiques conservées dans le sel ou placées avec soin dans l'alcool, ete., que M. Eschrieht recevait de lui, et qui ont fourni àce savant le sujet de tant de curieuses remarques anatomiques. C'est aussi grâce aux envois successifs faits par M. Holboll que plnsieurs des grands musées de l'Europe ont pu s'enrichir de pièees relatives aux Cétacés du Nord.

Ces données et celles qui avaient été réunies ả des époques plus anciennes, souvent par des observateurs placés à des distanees considérables les uns des autres, demandaient à être vérifiées et diseutées avec soin; la publication des doeuments restés inédits était également désirable : aussi avons-nous pensé que le moment était venu de reprendre dans son ensemble l'histoire des diffërentes familles de Cétacés.

Tout en insistant sur l'ostéographie de ees animaux, qui est 12 seule base certaine sur laquelle on puisse faire reposer leur classification et la distinetion de leurs différentes espèees, on ne négligera point, dans ee nouvel ouvrage, les autres côtés de l'histoire naturelle des Cèlacés.

Le nombre des espèees de ee groupe approche de deux eents, et de temps en lemps on en découvre encore de nouvelles qui avaient échappé jusqu'alors aux investigations des naturalistes. Nos mers, si fréquentées qu'elles soient, peuvent ellesmêmes en fournir. Témoin le Dioplodon europaeus, découvert il y a une vingtaine d'années à l'entrée de la Manche, et dont depuis lors il n'a été repris d'exemplaires sur aucun point du globe. Des espèees remarquables et qui servent de type à des genres particuliers n'ont elles-mêmes été observées qu'accidentellement, et plusieurs e sont eneore connues que par des pièces uniques, comme le Berardius Arnuxii, le Dioplodon densirostris et d'autres. Aussi ne saurait-on trop recommander aux navigateurs qui trouvent l'occasion de harponner de semblables animaux ou d'en recueillir des dépouilles même incomplètes, de les eonserver lorsqu'ils leur paraissent offrir quelque intérêt seientifique, afin de les soumettre à l'examen des savants en les déposant dans les collections publiques.

Des deux grandes divisions naturelles qu'il est facile d'établir parmi les Célacés, eelle des Célodontes ou Cétaeẻs pourvus de dents et sans fanons est la plus nombreuse en espèces et elle offre les caractères génériques les plus variés. Ses es-

\section{E-GUINEE}

ssus) Lawesii.

notrèmes de la famille

Touvelle-Hollande, mais

ne publić la description

appartient au Mĩusée de

ant aussi cette origine, ritamnique pour l'avanait à l'espèce, différente enait de signaler la prétte seconde espèce, qui e celle dont le Musée de ctc de l'Échidné d'Aus-

server doux des Échidnés

lles formes d'Lchidnidés nous tu sujet de l'histoire naturelle l'ostéographie des Monotrèmes de consacrer aux Mammifères et le troisième dans lequel il ieurement, dans le eourant de

(30 novembre 1877.)

sus (Echidna) proveniente dalla $v$. di St. nat. di Genova, t. IX, 
pèces arrivent à des dimensions très-diverses, et si les cachalots, qui en font partie, acquièrent le volume des balcincs, d'autres sont d'une taille déja moindre, comme les Hypérodons, les Ziphius, les Orques ou les Globiocéphales, ct il en est, comme les Marsouins, les Néoméris, les Pontoporia, etc., qui n'ont guère plus d'un mètre de longueur totale. Les Célacés constituant l'autre grande division de cet ordre de long ticètes ou Cétacés à fanons, animaux toujours gigantesques, moins variés dans leurs caractères que les Cétodontes, et dont on ne connaît qu'un moindre nombre d'espèces.

Les Cétodontes et les Mysticètes n'ont pas uniquement fourni des espèces à la faune actuelle. 11 en a aussi existé pendant la période tertiaire. On trouve des débris d’animaux appartenant à l'un et à l'autre de ces deux groupes dans les dépôts pliocènes ainsi que dans ceux que l'on a appelés miocèncs. Il est vrai que les terrains pliocènes ainsi que dans ceux que lon a appelés miocéncs. antéricurs n'en ont pas encore fourni. Les Cétacés d'espèces fossiles méritaicnt, auss bien que ceux qui habitent les mers actueles, aje fes faits nouveaux aux détals MM. Van Beneden et Paul Gerard.

que l'on a déja publies a cet èsard.

En ce qui concerne les espèccs vivantes, ils ne manqueront pas les occasions qui s'offriront à cux de donner, al sujet de lcurs mours, de leur synonymie et de leu nomenclaturc, ainsi qque de leur répartition hydrographique, des documents nouveaux qui compléteront ce qui sera dit relativement a leurs caractères anatomiques. A l'occa-
sion la capture de ces gigantesques animaux les occupera égalcment, puisqu'elle jette sion la capture de ces gigantè la manière de vivre des Cétacés, ct nous apprend le parti que quelque lumiere sur lanons, de leurs dents, de leurs os ou de leur huile, ct fournit de l'on tire de leurs fanons, de lansi qu'à l’indusirie.

Indépendamment des matériaux réunis à Paris et à Louvain, les auteurs du présent ouvrage ont pu utiliser ceux que l'on possède à Coponhague, à Londres, à Bruxelles, à Leyde, etc. Rien n’a été négligé pour rendre Jeur publication utile aux naturalistes a tux navigateurs, et e'est avec confiance que nous la soumettons au jugement du et aux navigateurs, et cest avec confa pas indigne de prendre place a côté du monument élevé a l'anatomie comparée par de Blainville, sous le titre d'Ostéographie des animaux vertébrés.

\section{CONDITIONS DE LA SOUSCRIPTION:}

L'ouvrage formera un fort volume in- $4^{\circ}$ de 500 pages avec figures sur bois, et accompagné d'un atlas de 50 planches grand in-folio lithographiées.

Il sera publié en 13 livraisons environ, renfermant chacune 5 feuilles de texte et 4 planches.

Prix de chaque liverison : 15 franes.

EN VENTE LES DEUX PREMIËRE LIVRAISONS.

PARTS. - IMPRIMERIE DE $\mathbb{M}^{\mathrm{m}}$ VEUVE BOUChARD-HUZARD, RUE DE L'ÉPKRON, 5. 


\section{CHAPITRE DEUXIÈME.}

\section{LES ÉCHIDNES DE LA NOUVELLE-GUINÉE}

Echidiza (Acanthoglossus) Bruijnii et Echidna (Tachyglossus) Lauesii.

On sait, depuis tries-peu de temps, qu'il existe des Monotrèmes de la famille des Ĺchidnés (1), non-seulement en Tasmanie et à la Nouvelle-Hollande, mais aussi à la Nouvelle-Guinée. MM. Peters et Doria ont mème publié la description de la tête d'une des espèces propres à cet archipel; elle appartient au Musée de Gênes (2), qui la doit à M. Bruijn, de Ternate.

M. Rolleston a parlé d'un animal du même groupe, ayant aussi cette origine, dans une communication qu'il a faite à l'Association britannique pour l'avancement des sciences, en 1877 ( de celle dont il est question ci-dessus, dont II. Ramsay venait de signaler la présence (5) dans les parties sud de la Nouvelle-Guinée. Cette seconde espèce, qui a reçu de M. Ramsay le nom d'Echidna Lawesii, diffère de celle dont le Musée de Gênes possède la tête osseuse, et quoiqu'elle soit distincte de l'Échidné d'Australie, on ne saurait encore l'en séparer génériquement.

J'ai pu, dans le courant du mois de novembre 187\%, observer deux des Échidnés

(1) L'intérèt tout particulier qui se rattache à la découverte de ces nouvelles formes d'Échidnidés nous a engagé à publier immédiatement les faits que nous avons pu réunir au sujet de l'histoire naturelle de l'espèce que nous avons pu observer et de son ostéologie. Le chapitre de l'Ostéographie des Monotrèmes que nous lui consacrons, est le second de ceux que nous nous proposons de consacrer aux Mammifères de la mème sous-classe. Le premier traitant de l'Échidné d'Australie et le troisième dans lequel il sera spécialement question de l'Ornithorhynque, ne paraîtront qu'ultérieurement, dans le courant de l'année 1878.

(30 novembre 1877.)

(2) W. Pelers et S. Doria, Descrizione di una nuova specie di Tachyglossus (Echidna) proveniente dalla Nrova Guinea settentrionale: Tachyglossus Bruijnii (Annali del Mrus. civ, di St. nat. di Genova, t. IX, av. 1 pl.; 3 décembre 1876).

(3) Proceed. Linn. Soc. New South Wales, t. II, p. 31, av. 1 pl.; 1877.

Nonotrèmes. 
du Nord de la Nouvelle-Guinée, l'un mâle, l'autre femelle, que M. Léon Lagglaize venait d'apporter à Paris et dont le Muséum a fait l'aequisition. Ce voyagreur se les étail procurés, avec le concours de M. Bruijn, à qui cette espèee de Monotrèmes est dédiée, dans les montağues des Karons (1), à une lıauteur de 1,150 mètres. Le corps du sujet femelle, que uous avons d'abord donné (2) comme étant le mâle, et dont le squelette est complet, était accompałsné de quelques parties détachées, dont une est la langue. Cet organe offre des caractères tout à fait particuliers.

Les naturels du pays où vivent les Échidnés de Bruijn, donnent à ces animaux le nom de Nokdiak; ils leur font la chasse au moyen de chięns qui les déeouvrent à l'odeur dans les terriers au fond desquels ils se liennent et grattent, en aboyant, le sol à l'endroit où ils ont reeonnu leur présence. On prend les Nokidiaks en pratiquant des trous d'environ un mètre de profondeur, ce qui donne accès dans leur retraite, et l'on mange leur chair. Les liabitants du littoral de la partie nord de l'Ile n'ont pas de nom pour désigner ces animaux; ils ne les connaissent même pas.

N'ayant pas vu l'Ėehidné dont M. Lamsay vient de donner la description et que je ne counais que par la Notice qu'il lui a consacrée, je ne m'occuperai dans ee chapitre que des deux exemplaires qui m’ont été cédés par M. Laglaize, et je les attribuerai l'un et l'autre à l'espèce de l'Acanlhoglossus Bruijnii.

Un résumé des observations auxquelles m'a conduit l'examen de ces deux Echidnés a été présenté par moi à l'Académie des sciences et publié dans le compte rendu de ses séances des $\because$ et 26 novembre 1877. J'y ai montré que ces animaux diffèrent par des caractères bien tranehés de ceux des Échidnés de l'Australie. Ils me paraissent devoir être distingués de ces derniers, non-senlement comme espèce, mais aussi comme genre, cequi oflre unintérêt particulier au point de vue de la géographie zoologique. An contraire, l'Echidna Lawesii, malgré la forme particulière de ses piquants, se rapproche davantage de l'Echidna aculeata et il a comme lui cinq doiggts onguieulés à cliaque pied. L'exemplaire entier et une lête isolée qu'on possède au Musée de la Nouvelle-Galles du Sud ont été rapportés par M. le R. Lawes, dont le nom a été donné à l'espèce nouvelle que ces pièces font connaître. Ils ont été recueillis au port Moresljy.

(1) MM. Peters et Doria citent le crâne dont ils ont parlé comme provenant du mont Arfak.

(2) Compt. rend. hebd., t. LXXXV, p. 837 et 990. 
Le nom d'Acanthoglossus (1), rappelant que la langue des Échidnés dont il s'git est garnie dans sa partie terminale d'aiguillons cornés, a été proposé par moi pour ce nouveau geure et j'ai cherché, en formant ce mot, à rappeler, au moyen de l'une des racines entrant dans sa composition, à lui donner une certaine ressemblance avec celui du Tachyglossus (2), qu'Illiger a substilué au terme d'Echidna, primitivement employé par Cuvier (5), el que plusieurs auteurs ont adopté en lui conservant le sens proposé par le grand naturaliste français. Il y aura ainsi deux genres dans la famille des Échidnidés : l'un sera celui des Echidna proprement dits ou Tachyglossus, l'autre, celui des Échidnidés différant à la fois des précédents par ses caractères extérieurs et ostéologiques, ainsi que par le pays qu'il habite; c'est ce genre que j’appelle Acanthoglossus; l'espèce propre au nord de la Nouvelle-Guinée, dnnt j'ai rappelé précédemment le nom, deviendra désormais le type de ce nouveau genre.

La ressemblance qui existe entre ce mot et celui d'Acanthoglossa, proposé il y a quelques années pour un genre de Coléoptères de la famille des lihynchophores, ne me paraîl pas devoir faire obstacle à son emploi. Il serait d'ailleurs facile de le remplacer si cetle manière de voir ne devait pas prévaloir; le nom de Proechidna ou tont antre pourrait alors lui être substitué.

\section{$\S 1$}

CarACtères extérieurs.

E Notre Echidné de la Nouvelle-Guinée (Acanthoglossus Bruijnii) est plus robusle et plus gros que ceux de l'Australic (Echidna aculeata ou $E$. setosa); il est en outre remarquable par l'allongement plus considérable de son rostre, au bout duquel est ouverte, de mème que dans l'espèce ordinaire, la bouche d'ailleurs petite comme chez cette dernière.

La longuueur tolale, qui ne dépasse guère $0^{\mathrm{m}}, 40$ dans l'Échidné australien, atteint ici un peu plus de $0^{\mathrm{m}}, 50$, et, sur cetle longueur, le rostre, c'est-à-dire la partie

(1) Axxv0x, épine; $\Gamma \hat{\lambda \omega} \tau \sigma \alpha$, langue.

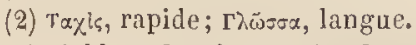

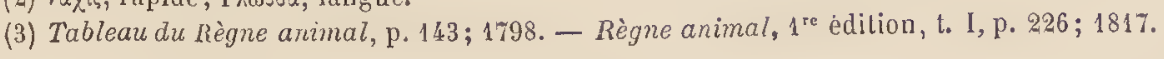


effilée de la tête, oeeupe $0^{\mathrm{m}}, \mathbf{1 5 0}$, en comptant depuis les yeux jusqu'à sa poinie, tandis que dans l'autre espèce, le bee n'a que $0^{\mathrm{m}}, 060$. Cet organe est noir et sensiblement arqué.

La queue est fort courte dans sa partie visible extérieurement; le tronc est robuste.

La eouleurn'est pas non plus la même. Au lieu d'être marron ou fauve roussâtre, elle est presque noire; les poils sont d'ailleurs plus doux et leur apparenee a quelque chose de velouté relevé d'une faible teinte plus claire approchant de la nuance eanelle. Quant aux piquants, ils n'ont pas non plus la même apparenee. Ils sont plus égaux entre eux et d'une foree pour ainsi dire intermédiaire entre les gros et les petits piquants de l'Éehidné australien; ils sont aussi moins nombreux et ceux de la région fessière ne sont pas autant rapprochés en faiseeaux; ell général, ils apparaissent eomme de petites pointes blanehes produisaut un effet assez élégant au-dessus de la partie soyeuse et souple du tégument et le front est muancé d'un peu de gris, tandis que le dessus des pattes tire au marron. L'Éehidné de Lawes a les piquants en grande partie aplatis et ses poils sont plus rares; sa eouleur est éģalement différente.

T'el est l'aspeet extérieur de l'exemplaire dont j'ai d'abord parlé, d'après les renseignements qui m'avaient été fournis, eomme étant le mâle, mais il est plus probable que e'est la femelle (1). Il manque d'éperons eornés aux talons, sa tête est plus grêle et son rostre un peu plus long que ehez l'autre sujet; il faut sans doute lui attribuer des mamelles, il est vrai séparées du eorps, qui m’ont été remises avec ces animaux.

L'exemplaire pourvu d'éperons cornés (2) a les piquants en grrande partie noirs, sauf pour quelques-uns dont la pointe passe au hlane. Sa tête osseuse se rapproche encore davantage par sa longueur moindre et ses proportions plus robustes de eelle dont MM. Peters et Doria ont donné la figure. On sait que les piquants du Tachyglosse sont forts et qu'ils sont pour la plupart jaunâtres avec la pointe noire.

Les ongles sont noirâtres: les antérieurs, droits et robustes; les postérieurs, longs et arqués en dedans. Mais ee qui a une plus grande valeur earaetéristique, e'est que ees ongles, d'ailleurs forts et disposés pour fouiller le sol, ne sont qu'au

(1) PI. VI, fig. 1.

(2) Pl. VI, fig. 7, et Pl. $1 \mathrm{X}, f i g .1$. 
nombre de trois aux pattes de devant comme à celles de derrière (1), tandis que l'Échidné déjà décrit a cinq doig̣ts onguiculés en avant et cinq en arrière.

La langue mérite aussi d'être mentionnée. Elle est ggrêle et beancoup plus longue que daus l'autre genre; elle mesure, en effet, de sa partie basilaire à sa pointe, $0^{\mathrm{m}}, 210$, au lieu de $0^{\mathrm{m}}, 083$. Les papilles cornées de sa base ont aussi une autre disposition; elles sont réunies au nombre d'une soixantaine sur la face supérieure de la partie postérieure de cet organe et y forment un groupe de petites épines rudes au lieu d'un amas confus de simples saillies; on ne voit pas au devant de cet amas les quatre lamelles transversales frangées des Échidués tachyglosses. La langue entière ressemble à un long ver, annelé dans sa partie moyenne; elle n'a pas l'apparence acuminée qu'elle présente dans l'autre genre et, au lielı d'être lisse et douce dans le reste de sa longneur, elle est disposée en gouttière vers sa pointe on portion antérieure, et cette gounttière présente trois séries d'épines cornées dirigées en arrière, dont deux marginales et la troisième médiane. Les muscles sternoglosses, dont la portion attenante à la langue a été conservée, doivent offrir un développement considérable.

On voitsur la membrane du palais, depuis la partie la plus rapprochée des arrịere-narines jusqu'à celle qui recouvre le trou incisif, des saillies cornées, d'abord rangées sur cinq lignes ou séries transversales, dont la première et la seconde se composent de tubercules de forme à peu prês conique plus nombreux que ceux des troisième et quatrième et surtout que ceux de la cinquième qui n'en compte plus que quatre. Les deux groupes suivants en possèdent chacun deux, mais qui sont presque confluenis. Viennent au delà deux tubercules simples et coniques, situés à une distance à peu près égale à celle des tubercules en groupes. Ensuite, ils redeviennent bigéminés ou d'une complication un peu plus grande. Ils semblent être particulièrement en rapport avec les épines cornées de la langue et paraissent destinés à faciliter la préhension des aliments. La disposition de ces épines ou tubercules colnés du palais est à pen près identique dans nos deux exemplaires. On sait, d'aulre part, que le palais de l'Échidné australien ne présente que sept rangées sériales d'épines serrées les unes contre les autres; elles sont peu différentes de celles qui se voient à la partie postérieure de la membrane palatine de

(1) Pl. VI, fig. 5-6. 
I'Acanthogglosse (1), bien qu'elles ne soient pas en même nombre ct que leur apparence ait quelque chose de particulier.

\section{$\S$ II}

SQUEILTTE.

Si l'on compare le crâne de notre Échidné acanthoglosse à celui du Tachyglosse ou Échidné de l'Australie, on y remarque plusieurs traits distinctifs justifiant la séparation de ces dcux sortes d'animaux comme espèces distinctes l'une de l'autre et dont quelques-uns contribueront aussi à caractériser le genre que j'ai proposé d'établir pour y placer le premier d'entrc eux.

Lin cffet, dans cclui-ci, non-seulement le cràne (2) acquiert une longueur presque double $(0,20$ au lieu de 0,11$)$, mais il est arqué au lieu d'être droit et aplati à sa facc inférieurc; en même temps son rostre ou portion faciale, dont la courbure cst plus accentuée quc ccllc de la loge contenant le cerveau, est proportionnellement heaucoup plus prolongée. La surface palatine cn est plus excavée, par suite du rclèvement des bords latéraux de la mâchoire, et elle ressemble davantage à une gouttière. Elle est aussi relativement plus étroite, que l'on considère soit la région ptérygo-palatine, soit la partie fournie par les maxillaires, ou bien encore l'espace occupé par les intermaxillaires depuis leur implantation dans la fissure antérieure du bord extcrnc des maxillaires jusqu'à leur réunion en avant de l'ouverture extérieure des narines. L'échancrure qui existe entre les ptérygoïdiens ne forme que la moitié d'un ovale et elle ne se prolongc pas entre les palatins comme elle lc fait dans l'Échidné connu antérieurement où elle figure un triangle isocèłe à sommet fort aigu. L'arc supérieur du trou rachidien cst aussi plus régulicr, et l'on n'y voit pas la petite échancrure ovalaire qui le surmonte dans l'autre genre d'Échidnés. La boîtc cérébrale est en mèmc temps plus ample ct le moule de sa cavité in térieure montre qu'il existe une plus grande complication des circonvolutions propres aux hémisphères cérébraux. Elles y sont plus nombreuses pour chacune des divisions de la surface des hémisphères et partout un peu

(1) Pl. VII, fig. 5 .

(2) PI, VI, fig. 1, $1 a, 16$ et PI. IX, fig.1 
plus 'largies; c'est ce que l'on reconnaitra en comparant la figure que j'en donne ici (1) avec celle du moule cérébral du Tacliyglosse que j’ai publiée précédcmment (2). C'est là une disposition qui se trouve souvent dans les Mammifères d'un même groupe, si l'nn passe d'un genre dont les espèces ont une plus petite taille à un autrc dans lequel elles sont plus grandes; j'en ai fourni de nombreuses preuves dans mes Mémoires relatifs aux formes cérébrales de différents ordres de cette classe (5).

D’ailleul's les principales particularités signnalées par les anatomistes qui sc sont occupés de l'Éeliidné ordinaire, Cuvier (4), M. Owen (5), cte., s'observent aussi dans cclui qui nous occupe en ce moment, et l'on y trouve une nouvelle confirmation de l'opinion que plusieurs d'entre eux s'étaient déjà faite lorsqu'ils ont comparé la famille dont il est devenu le type avec les Ėlentés, plus particulièrement avec les Fourmiliers et les Pangolins, animaux dont les Échidnidés semblent, en effet se rapprocher par les principaux traits de leur ostéologie. On se rappelle que ces affinilés n'avaient pas échappé au naturaliste anglais Shaw, l'un des premiers autenrs qui aient parlé de l'Éehidné ordinaire, puisqu'il en avait fait, ainsi que nous avous déjà eu l'occasion de le rappeler, une espèce de Fourmiliers sous le nom de Myrmecophaga aculeata.

Les cornets etlımoïdaux forment, dans la partie antérieure de la cavité crànienne, une masse élargie, compliquée et relevée; ils sont placés en avant d'une sorte de selle turcique qui se continue en unc large gouttière au-dessus des os sphénoïdes

L'orifice extérieuc des narines est plus régulièrement ovalaire et plus gurand.

Le tron incisif est au contraire de mème forme ou à peu près.

Bien que le crâne d'Échidné de la Nouvelle-Guinée que nous décrivons, ct qui est celui de notre exemplaire fenılle, soit dans un état très-avancé d'ossification et que presque tous les os en soient devenus coalescents, on y remarque les principaux caractères connus dans l'autre genre d'Échinidés et la disposition des trous nerveux et vasculaires y est sensiblemeut la même. Le crâne dı mâle (6) donne lieu

(1) Pl. VII, $f i g .3$.

(2) Nour. Arch. Mus., t. V, p. 248, Pl. XIV, fig. 16.

(3) Nouvelles Archives du Museum et.Journal de Zoologie.

(4) Oss. foss., t. V, part. 1, p. 145.

(5) Todd's Cyclopedia of Anatomy and Physiology (article Monolremata).

(6) P]. IX, fig. 1. 
aux mêmes remarques; mais, ainsi que nous l'avons déjà dit, il est un peu plus court que cclui de la femclle $(0,175$ au lieu de 0,20$)$, est plus robuste et a le rostre moins cffilé.

Le trou sous-orbitaire ( 1 ) est également fort allongé $(0,100)$; il se eontinue à peu près jusqu'à l'alignement du bord postérieur de l'orifiee osseux des narines externes et débouehe de ee eôté de la tête, à 0,022 environ de la pointe du bec, pár deux orifiees, l'un allongé, situé sur le bord externe du rostre, l'autre plus court et presque marginal.

On voit aussi extćrieurement sur les côtés de la boîte ećrćbrale, à partir d'un point située à 0,008 au-dessus de la cavité glénoïde, jusqu’à un autre point enfoncé sous le commeneement de la fosse sphéno-orbitaire, en avant de la même cavité, lc canal particulier à cettc famille de Monotrèmes qui est ouvert à la face interne d'un os que Cuvicr regardait eomme ćtant le temporal. Ce canal (2) file entre cet os et le parićtal; on le retrouve aussi ehez l'Ornithorhynque, mais il y cst trèseourt, et Meekel le fait passer entre les deux raeines postérieures du zygomatique, ce qui cst aussi la manicre de voir de M. Owcn, pour qui l'os dont il s'agit est en cffet le véritable zygomatique. Quant au canal lui-même, il reçoit chez les Échidnidés, ainsi que nous l'avons vérifié, une branche artérielle, fournie par la carotide cxterne, qui cnvoie des rameaux dans les os reeouverts par la plaque zygomatique dont nous venons de parler, et jusque dans le rostre où ils pćnc̀trent par le frontal.

Le cadre du tympan (5) est appliqué, de mème que eelui de l'Échidné australieı, autour de la grande eupule existant entre le ptérygö̈dien ct le temporal, cupule qui répond à l'oreille moycnne et forme la cavité de la caisse. Il cn marque la limitc extćrieurc et le tympan en borne l'étendue du même côté. La fente osseuse qui log̣e la trompe d'Eustaclıe y aboutit de même cn constituant une rainure placće cntre lc ptérygoïdien et le mastoïdien; mais eclte rainure n'a pas tout à fail la mème apparence dans les deux genres. Le cerele tympanique est aussi plus grand, plus fort et plus large dans l'Acanthoglossus, cı le marteau, dont l'aspect cst un peu difiérent, envoie, après avoir fourni son manche, c'est-à-dire l'apopliyse par laquelle il

\footnotetext{
(1) Pl. VII, fig. $1 a^{*} \mathrm{a}^{*}$

(2) Pl. VII, fig. $1 b^{*}$ à $*$

(3) Pl. VII, $f g$. 4 el $4 a$.
} 
s'applique sur la membrane du tympan, un long prolongement qui contourne extérieurement l'arc correspondant du cadre tympanique et s'étale en avant de lui en un flabellum plus large et plus développé que cela n'a lieu chez le Tachygosse; aussi semble-t-il que l'on doit y voir un reste ossifié et d'une étendue plus considérable que d'habitude de la branche par laquelle cet élćment de la chaine auditive, réduit chez les Mammifères adultes à sa partie constituant la première pièce de la chaîne des osselets de ces animaux, c'est-à-dire le marteau, est en rapport cliez le foetus avec le cartilage de Meckel. On sait que ce cartilage est considéré comme étant l'un des éléments constitutifs de la mâchoire inférieure pendant la vie embryonnaire. Le cercle tympanique et le marteau de l'espèce d'Échidnidés dont nous parlons devront donc être pris en considération lorsque l'on discutera, comme l'ont fait M. Peters (1) et M. Huxley (2), la signification anatomique des mêmes pičces. C'est ce que l'on a déjà fait remarquer à propos du Tachyglosse et c'est ce que justifie la condition inférieure de ces deux genres qui sont, avec l'Ornithorhynque, les Mammifères les plus rapprochés des ovipares.

La mâchoire inférieure, un peu moins arquée que la supérieure, a ses deux branches symphysées sur la moitié environ de leur longueur au lieu d'ètre disjointes, mais cette symphyse n'est pas ossifiée.

Quant à la mảchoire elle-même, elle est proportionnellement plus robuste; son condyle est plus marqué et la saillie qui répond à l'apophyse coronoïde plus forte et plus rejetée en dehors. L'apophyse angulaire y est aussi moins éloignnée du condyle, qui a plus de force, et l'on y voit très-distinctement le canal dentaire dont l'ouverture est à la face interne des branches mandibulaires, opposé à la saillie coronoïde.

Il existe aussi chez l'Acanthoglosse un trou mentonnier très-apparent, situé au commencement du tiers terminal, et qui se continue en avant sous la forme d'une rigole.

Ces particularités méritent d'être rcmarquées, attendu qu'elles indiqucnt de nouvelles différences avec I'Échidné tachygglosse, et si l'on fait attention au facies

(1) Monatsbericht Berlin Acad, wissensch., 1863, p. 598, P1. I.

(2) Proceed. zool. Soc. London, 1869, p. 391. 
de la tête osseuse de l'Aeanthoglosse, ainsi que de sa mâchoire inférieure, on est frappé des ressemblances qu'il présente sous ce double rapport avec les mêmes parties du squelette envisagées dans l'Aptéryx.

Mais cette similitude remarquable s'observe entre l'aspect général du crâne de l'Acanthoglosse et eelui des Aptéryx, qui sont de singuliers oiseaux particuliers à la Nouvelle-Zélande, sans que pourtant les principaux caractères, de même que eeux du cråne du Tacliyglosse, cessent de rester conformes à ceux des Mammifères, envisagés comme elasse.

C'est ce que l'on constatera si l'on envisage le nombre total des os craniens de ces Monotrèmes, l'absence ehez eux de vćritable os carré, l'existenee d'un condyle articulaire de la mâchoire inférieure, si réduit qu’il soit, la conformation de leur palais et la présenee de deux condyles occipitaux au lieu d'un seul, eomme cela se voit ehez les oiseaux et chez les reptiles de tous les ordres.

L'Acanthoglosse n'a pas le même nombre de vertèbres que l'Éehidné australien ou Tachyglosse; il possède 17 vertèbres dorsales ou costifères au lieu de 13 et peut-être une caudale de plus. A part eette différence, il s'éloigne à faiblement de l'espèce d'Australie par la forme de ces pièces du squelette, mais elles sont plus grosses ehez lui, ce qui`est en rapport avec le volume plus considérable de son eorps; elles ont la même apparence générale et les faces par lesquelles leurs centrums sont en rapport entre eux sont de même très-légèrement excavées. Nous donnons des figures de ees différentes vertèbres, savoir : l'atlas, l'axis, dont l'arc supérieur est plus long et a sa face articulaire postérieure plus exeavée, les cervicales 5 à 7 , les première et dix-septième dorsales ainsi que l'ensemble de cette même région, les lombaires, une partic des sacrées et les eoceygiennes (1).

Par suite de l'état plus eomplet de l'ossification, les trois sacrées postérieures, qui constituent en réalité le véritable sacrum, attendu qu'elles tiennent toutes trois à l'os des iles, ont leurs apophyses épineuses soudées en une crête continue qui s'élève au-dessus de la synostose qu'elles constituent par leur réunion, au lieu de rester indépendantes les unes des autres, comme elles le font chez le Tachyglosse.

De mème que les vertèbres et toutes les autres pièces du squelette, les eôtes sont à leur tour plus robustes que dans l'espèee australienne. Cependant la der-

(1) Pl. VIII et IX. 
nière, qui est la dix-septième, reste beaucoup plus petite que les autres et sous ce rapport ellc cst très-inférieure à la dernièrc, c'est-à-dire à la quinzième, de l'Échidné proprement dit. Ces côtes sont également ossifiées aussi bien dans leur partie appelée chondropleure, qui reste si souvent cartilagincuse chez les autres mammifères, que dans leur partie osscuse ou ostéopleure; mais il existe entre ces deux moitiés de chacune d'elles un intcrvalle cartilagineux, long de $0^{\mathrm{m}}, 010$ environ, destiné à faciliter les mouvements de dilatation de la cage thoracique; c'est là une disposition rappelant ce qui existe chez les Crocodilcs et les Sauriens. Les six premières côtes sont vertébro-sternales; elles ont leurs chondropleures à peu près cylindriques; celles qui suivent sont aplaties et comme imbriquées les uncs sur.les autres dans leur partie ventrale ou chonchropleurique, qui diminue d'étendue, au fur et à mesure que l'on s'éloigne des côtcs vertébro-stcrnales, et la dernic̀re paraît en manquer à pcu près complétement.

La première piècc du sternum (1) a sa saillie antérieure prolongée, comme cela a lieu chez les autres Monotrèmes, par un os en forme dc T dont la tige verticale présente ici une forte carêne située près de sa base; celle-ci est elle-même resserrée obliquement entre le bord interne des coracoïdiens (2) gui ne sont pas symétriques entre eux et la partie principale des mêmes os se soude de même intimement, du moins dans les sujets adultes, arec la portion glénoïdienne des omoplates. Les claviculcs n'offrent rien de particulier', si ce n'est que, dans notre exemplaire, elles sont déjà intimement soudées aux branches transversales de l'os en 'I. Enfin les omoplates sont grảndes et fortes, et leur épine est également peu sentie; leur col parait un peu moins dégagé et l'angुle formé par la réunion de leurs bords susépineux n'a pas la mème inflexion.

Après la première sternèbre, portant l'os en $\mathrm{T}$, on en compte six autres suivies à leur tour d'un appendice xyphoïde composé de six segyments osseux alternativement plus grands et plus petits. L'appendice xyphoïde du Tachyglosse reste presque entièrement cartilagincux; sa base seule parait s'ossifier.

Etudions maintenant les os des membres (3).

(1) Pl. VIII, fig. 8 et $8 a$.

(2) Ou plutôt des épicoracoïdiens de Guvier, pièces dont nous discutons la signification en parlant de l'Échidné de la Nouvelle-llollande.

(3) Pl. VIIl, fig. 10-13, et Pl. IX, fig. 8-10. 
L'humérus est comme tordu sur lui-même dans la partie moyenne de sa diaphyse, laquelle est beaueoup moins forte que ses extrémités où il s'élargit.

La direction de ees dernic̀res est, de son eôté, différente pour chacune d'elles: celle d'en haut est exeavée à sa faee antérieure, eelle d'en bas est irrégulièr'ement aplatie; et l'os y présente aussi cu dehors du trou de sa large épitroehlée une poulie articulaire dont la saillie, de forme demi-globuleuse un peu ovalaire, est placée entre le fort épicondyle et l'épitroehlće, celle-ci faisant saillie en dehors, de manière à dépasser de beaucoup la partie montante de l'os. L'épitroehlée oeeupe une large superficie demi-eireulaire, en dedans de laquelle est le trou épitrochléen, dont la direction est oblique transversalement. La erêtc, qui lui fait suite, longe la partie moycnne de l'os en formant unc rentréc curvilignc; elle va rcjoindre la tubćrosité supérieure interne, tandis que la erête deltoïdienne deseend sur le milieu de l'os, partant de la tubérosité externe, et forme sa earêne antérieure. Lcs deux extrémités sont diriguées à angule droit l'une par rapport à l'autre. Ce sont là d'ailleurs des caraetères propres à l'Éehidné australien, mais qui sont iei bien plus aceentués.

Il n'y a guc̀re à mentionmer, pour l'avant-bras, que le rapprochement plus intime des dcux os qui le constituent, leur forme restant à peu près la mêmc; mais ils ont toujours plus de force.

La ressemblanee est moindre pour la première rangée des os du carpe (1).

Ainsi le scaphoïde et le lunaire ne sont pas réunis par synostose, du moins du eôté droit, ehez le sujet décrit (2); le radius appuie sur tous les deux à la fois comme il lc fait, du reste, dans le Taehyglosse, sur l'os unique qui les représente.

Le pyramidal cst moins épais que ehez ce dernier, mais il porte de même à sa face postérieure le pisiformc, qui est à peu près globuleux.

Le trapèze est petit, ce qui est en rapport avee l'état incomplet du pouee.

Le trapézoïde, le grand os et l'os crochu ont plus de force; cc dcrnier porte aussi le métaearpien du quatrième doiggt ainsi que eelui du einquième.

Les trois métacarpiens intermédiaires, qui portent seuls des doights pourvus d'ongles, sont robustes et de forme carrée; le premier et le cinquièmc ont un moindre volume.

(1) Pl. VIII, fig. 12.

(2) Ceux du côté gauche sont, au contraire, réunis comme dans les Échidnés australiens. 
La première phalange des doiggts intermédiaires est plus petite que la scconde et surtout que la troisic̀me, laquelle est en amande allongée, tronquée à sa base.

Le pouce n'a qu'une phalange; le petit doigt n'en a que deux.

A la face inférieure du carpe, existe un grand sésamoïde, irrégulièrement rectangulaire comme il y en a dans le Tachyglosse et dans beaucoup d'autres Mammifcres, certains 'Tatous, par excmple (1).

Si nous passons au bassin, nous ne constatons pas de grrandes différences. La première vertèbre sacrée, que l'on pourrait aussi appcler la dernic̀re lombaire, porte bien sur l'os des iles par ses parties latérales, mais elle ne fait pas aussi complétement corps avec la seconde que les trois suivantes lc font enscmblc; en outre, elle n'adhère pas très-fortement au bord supéricur de l'os des iles comme cela a lieu pour ces dernières. La cavité cotyloüde est aussi percée d'un trou arrondi ct le trou obturateur est à peu près de même forme que chez les Échidnés d’Australie.

Le fémur est grrêlc, long et rappelle, jusqu'à un certain point, celui du Lestodon, grand Édenté fossile, propre à l'Amérique méridionale, dont j'ai donné ailleurs la description (2). Sa crête interne est plus tranchante et l'externe plus saillante dans son milieu que dans le Tachyglosse, ce qui semble indiquer un commencement de troisième trochanter.

Le grand ct lc petit trocliantcr sont plus accentués ct il existe sur la face antérieure du même os, au-dessus de la poulie rotulienne, une dćpression constituant une sorte d'excaration au lieu d'un sillon transversal.

Les deux os de la jambe ne different guc̀re que par leur volume, quoique la saillie qui remonte de l'extrémité supéricure du péroné soit plus élevée et plus sécuriforme dans son apparence générale.

L'astragale présente la même conformation que cliez le Tachygglosse; il ne porte pas, dans le sujet dont nous donnons la description et que tout nous porte à considérer comme étant du sexe femelle, les deux articles constituant l'appareil corné des Échidnés mâles de l'espèce australienne; mais cet appareil se trouve dans l'autre excmplaire.

Dans le sujet pourvu d'un éperon corné, nous retrourons l'insertion de cet or-

(1) Voir P. Gervais, Remarques ostéologiques au sujet des pieds des Édentés (Journal de Zoologie, t. IV p. 79, Pl. II-V).

(2) Mém. Soc. géol. France, $2^{\circ}$ série, t. IV, p. 29, Pl. XXVI, fig. 1. 
gane sous la peau et il va jusqu'à l'astragale, sur lequel il s'insère par une pièce basilaire irrégulière et aplatie, et, par suite, assez diffẻrente par sa forme de celle qu'elle a dans le Tachyglosse. Sa pointe cornée est presque semblable et il est à peu près aussi fort que dans l'autre genre; sa couleur est blonde (1).

Le scaploïde est plus globuleux et les os de la seeonde rangée tarsienne ou os du mésotarse sont les trois eunéiformes, dont le second est le plus petit, et le cuboide, ce qui rentre dans les conditions ordinaires. Le cuboïde, assez allongé, va du seaphoïde au métatarsien du cinquième orteil et il porte latéralement par sa partie supérieure d'un côté sur le scaphoïde, de l'autre sur le calcanéum qui forme une grosse tubérosité digitiforme rabattue en avant. Ces dispositions ne sont pas aussi prononcées dans l'espèce ordinaire.

Trois orteils seulement portent des ongles; ee sont les trois intermédiaires (2). Le pouee n'a qu'une seule phalange, mais elle est plus volumineuse que celle du doigt eorrespondant de devant. Le cinquième orteil en a deux, également moins réduites que leurs eorrespondantes du pied antérieur. Les plıalanges onguéales des deuxième à quatrième orteils sont longues et aiguës.

\section{EXPLICATION DES PLANCHES VI A IX.}

(Acanthoglossus Bruïnï.)

\section{PLANGHE VI.}

Fig. 1. Échidné de la Nouvelle-Guinée, femelle vue de profil.

Fig. 2. Partie terminale du rostre, vue en dessus, pour montrer les narines.

Fig. 3. Partie terminale du rostre, vue en dessous, pour montrer la bouche.

Fig. 4. Patte de devant, vue en dessous.

Fig. 5. Patte postérieure, vue en dessous.

Fig. 6. Piquants : $a$ du mâle; $b$ de la femelle; $c$ et $c^{\prime}$ de l'Éehidné d'Australie.

(1) Pl. IX, fig. 11.

(2) Dans l'Échidné de Lawes, qui a cinq doigts, comme celui d'Australie, le second orteil est sensiblement plus long que les autres, ce qui se voit aussi chez celui-ci. 
La $f g .1$ cst réduitc à $\frac{1}{2}$; les $f i g .2$ à 6 sont de grandeur naturelle; celles des numéros 2 à 5 sont tirées de l'exemplaire mâle. Les coupcs des piquants, montrant la gaîne et la moclle qu'elle renferme, sont tirées de l'Acanthoglosse (7) ct du Tachyglosse (7a); elles sont très-grossies.

\section{PLANGHE VII.}

Fig. 1. Le crâne de l'Acanthoglosse femclle, vu de profil : * marque les orifices de sortie du trou sous-orbitaire.

Fig. 1a. Le même, vu en dcssus.

Fig. 16. Le même, vu en dessous. Les signes* et * indiquent l'entrće et la sortie du canal temporal.

Fig. 2. La mâchoirc inférieure, vuc par sa face cxterne.

Fig. 2a. La même, vue en dessus, c'cst-à dire par sa facc linguale.

Fig. 3. Moulc intra-crânien, donnant la disposition des circonvolutions du cerveau.

Fig. 4. Le ccrcle tympanique, la membrane du tympan ct lc marteau, vus par la face externe.

Fig. La. Les mêmes, vus par la face interne.

Fig. 5. La menbrane du palais, pour montrer les saillics cornées dont clle est armće.

Fig. 6. La langue, vue cn dessus, pour montrer la partie des muscles sternoglosses à laquelle elle donne attache, les pointes accumulées à sa base, les tubercules cornés insérés sur les deux premiers tiers de sa longueur ct les épincs, disposées sur trois sćries longitudinales, qui arment sa dernière partic.

Ces figures sont toutes de grandeur naturelle, sauf les $/ \mathrm{g}$. 4 et $4 a$. qui sont au double, et la fig. 6, qui cst aux $\frac{2}{3}$; clles sont tirées de l'exemplaire femelle.

\section{PLANCHE VIII.}

Fig. 1 ct 1 a. L'Atlas, vu par scs faces antérieure ct postérieure.

Fig. 2, $2 a, 2 b$ ct $2 c$. L'axis, vu de profil, en dessous, en avant ct par sa face postérieure.

Fig. 3. La troisièmc vertèbre ccrvicalc, vue en dessous.

Fig. 3a. La même, vuc par la facc postérieure.

Fig. 4. La quatrième vertèbre cervicale, vue en dessous.

Fig. 5. La cinquième vertc̀brc cervicale, vue en dessous.

Frg. 6. La sixièmc vertèbre cervicalc, vue en dessous.

Fig. 7. La septièmc vcrtèbrc cervicalc, vuc en dessous.

Fig. $7 a$. La mêmc, vue par sa face postérieure.

Fig. 8. La ceinture scapulaire, vue par sa face antérieure, pour montrer les rapports de la clavicule, du coracoïdien et de l'omoplate. On voit aussi l'os cn T, aux branchcs horizontales duquel lcs clavicules sont soudées, et les dcux pièces épicoracoïdicunes.

Fig. $8 a$. Ccs deux dernières pièccs, vucs en arrièrc, ainsi que les deux pièces allant à l'os en T qu'elles supportent.

Fig. 9. Le sternum ct les chondroplcures ici osseux.

On voit, pour quelques-unes des côtes, Ia courte portion cartaligincuse qui unit les chondropleures ou 'portion habituellement cartilagincuse des côtes aux ostéopleures ou partie vertẻbrale de ces appendices.

Fig. 10. L'humérus, vu par sa face antérieurc. 
Fig. 10a. Son extrémitć supérieure, vue du même côté.

Fig. 10b. L'humérus, vu par sa face postérieure.

Fig. 10c. Son extrémitć supérieure, vue en arrière.

Fig. 11. L'avant-bras (radius et eubitus), vu par sa face externe.

Fig. 11a. L'avant-bras, vu en avant.

Fig. 11b. L'avant-bras, vu par sa face interne.

Fig. 11c. L'avant-J)ras, vu par sa face postérieure.

Fig. 12. La patte antérieure, vue en dessus; eette figure est tirée du pied dont le seaphoĩde et le semi-lunaire sont séparés l'un de l'autre; ces deux os sont, al contraire, réunis en un seul, au pied du côté opposé, ee qui est le cas ordinaire pour l'Échidné d'Australie.

Fig. 12a. La méme, vue en dessous; on a représenté sur cette figure les sésamoïdes placés audessous des phalangines des trois doigts principaux.

Fig. 13. Le sésamoĩde palmaire, vu séparément en dessus et en dessous; au double de la grandeur naturelle.

Ces figures sont toutes de grandeur naturelle; elles sont tirées de l'exemplaire femelle.

\section{PLANCHE IX.}

Fig. 1. Crâne de l'exemplaire mâle, vu de profil.

Fig. 2. Les vertèbres dorsales et la partie vertćbrale des côtes, vues en dessus.

Fig. 3. La première vertèbre dorsale, vue en avant.

Fig. 4. La dernière vertèbre dorsale, vue en arrière.

Fig. 5. La région lombaire, vue par la face supćrieure.

Fig. 6. Le bassin et les os marsupiaux, vus en avant.

Fig. 7. Lcs vertèbres caudales, vues en dessus.

Frg. 8. Le fćmur, vu par sa face antérieurc.

Fig. 8a. Le fémur, vu par sa face postérieure.

Fig. 9. La jambe (tibia et peroné), vue par sa face extérieure.

Fig. $9 a$ La même, vuc par sa face postéricure.

Fig. 10. La patte de derrière, vue en dessus.

Fig. 10a. La même, vue en dessous.

Fig. 11. L'éperon eorné de la jambe du mâle et les deux os qui les soutiennent.

Ces figures sont toutes de grandeur naturelle; sauf la première et la onzième, elles sont tirées de l'exemplaire femelle. 

EXPLORATION GEOLOGIQUE A LA MER MORTE, EN PALESTINE ET EN IDOMEE, par Louis Lartel, docteur ès sciences. Un trèsfort volume grand in-4. $4^{\circ}$, avec figures dans le texte, accompagné de grandes cartes, panoramas et coupes gèologiques imprimées en plusieurs couleurs, et de planches paléontologiques et stratigraphiques lithographiées.

$40 \mathrm{fr}$.

Esquisse geologique et paléontologique. Aspect physique de l'Orjent. Recherches géologiques faites jusqu ho co jour. Massifs cristallins et selisteux. Roches éruptives. Description des grès et du terrain stratigraphique, du terrain de craie. Terrains crétacés. Paléontologie du terraln de craie: vertébrés, annelés, mollusques, radiaires, protozoaires. Tcrrains tertiaires. Terraln quaternaires. Terrains voleaniques. Terrains contemporains et phénomèaes auxqueis est due leur formation. Vestiges đes temps préhistoriques. Dolmens et menlirs. Formation du bsssin de la mer Morte. De l'hypothèse de l'ancien prolongement du Jourdain jusqu'à la mer Rouge et d'une ancicnne communication marine de la mer Morte avec les mers environnantes. Théories diverses émises ì propos de l'origine de la mer Morte. Formation et origine du lac lui-même, salnre et variations successives de son niveau. Analyses ehimiques qui en ont été faites jusqu'à ce jour. Recherches snr la composition de l'eau en divers points de sa surface, ainsi qu'à différentes profondeurs. Instruments employés dans le puisage. Résultats fournis par l'analyse chimique des caux recueillies en différcnts points et iे direrses profondeurs. Tableaux numériqucs de comparaison. Scls contenus dans les affiuents de la umer Morte. Origine probable de la salure de eette mer intérieuro. Émanations bitumincuses de la mer Morte et de la Judée.

PLIS GÉRÉBRAUX DE LHOMME ET DES PRIMATÉS (MÉMOIRE SUR LES ), par M. P. Gratiolel, chef des travaux anatomiques au muséum d'histoire naturelle. 1 vol. in-4" accompagné d'un atlas in-folio de 13 planches (épuisé).

$45 \mathrm{fr}$.

ASTRONOMIE, HYDROGRAPHIE ET PHYSIQUE des Voyages en Yslande, Scandinatie, Laponie, au Spitzkerg el aux Féró. par M. Bravais, lieutenant de vaisseau, professeur à l'Ĺcole un atlas de 31 planches grand in-folio.

$180 \mathrm{fr}$.

On vend séparément :

Astronomie, Hydrographie et Marées. 1 vol. in-8 accompagné d'un atlas de 9 planches in-fol.

$40 \mathrm{fr}$

Longitudes et latitudes déterminées. - Marées observécs. - Dépression de l'horizon et phẻnomènes dil mirage. - Sur les températures de la mer. - Sondages et courants dans les mers du

Magnètisme terrestre. 3 vol. gr. in- 8 avec un atlas de 8 planches in-folio.

$60 \mathrm{fl}^{\circ}$.

Variations ct mesure de la décllnaison magnétique, ainsi que l'intensité magnétique

Mètéorologie. 3 vol. gr. in -8 avec un atlas de 6 planches infolio.

$60 \mathrm{fr}$.

Observations météorologiqnes faites à tcrre pendant les relâchea et pendant l'hí vernage. - Comparalsons bsrométriques faites dans le nord de l'Europe. - Varia tions et état moyen du baromètre. - Sur la température de l'aír, ses variations son état moyen. - Des températures par rayonnement. - Hygrométrie. - Nuage mique.

Aurores boréales. 1 vol. gr. in -8 avec un atlas de 12 planches gr. in-folio.

$40 \mathrm{fr}$

Deription de toutes les observalions arec leurs résultats.

Ouvrage publie par ordre de M. le Minislre de la marine.

OBSERVATIONS HYDROGRAPHIQUES ET PHYSIQUES recueillies par M. Duperrey pendant son voyage autourdu monde sur la
corvette la Coquille. 3 vol, in $4^{\circ}$ et atlas grand in-folio. $250 \mathrm{fr}$.

Hydrographie. 1 vol. gr. in-folio composé de 52 cartes et 12 feuilles de texte.

$200 \mathrm{fr}$.

Physique. 1 vol. in- 4 de 261 pages, 7 planches, dont 6 cartes. Hydrographio. 1 vol. in-4. de 164 pages. - Hydrographie et Physique. 1 vol. in-4 de 133 pages.

Tous les savants connaissent les travanx si justement estímés de M. Duperrey sur
pole nord et l'intensité magnétiquo; c'est le seul ourrage oì 1ls se trouvent consignés. Ouvrage publié par ordre de $M$. le Ministre de la marine.

HISTOIRE DE LART EGYPTIEN D'APRES LES MONUMENTS, depuis les temps les plus reculés jusqu'à la domination romaine, 1 volume de texte.

Ges 2 volumes se composeront de 40 livraisons, renfermant chacune 4 planches sur papier grand raisin ia-plano, exécutées en chromolithographie par les meilleurs artistes, et d'un volume in-4 de texte.

The premier volume est consacré naiquemen a l'architecture polychrome des Égyptiens.

Le secona volume est consacré au dessin, à la peinture, à la sculptare et à l'art industriel de ce peuple, qui a précédẻ tous les autres dans la carrib̀re des arts, ct qul témoigne d'uno cirilisation très-arancée.

Le rolame le texte, grand in-4, orné de nombrenses vignettes, forme une histoire complete de l'art égyptien, qui est mis en parallele avec l'art assyrien, lindou, ftropien et grec. L'listoire éfyptienne est ainsi rattachẻe à celle des civilisations contemporaines, et le lecteur peut suivre la filiation et le développement de la penséo artistique chez les différentes races de l'ancien monde.

En vente : les trente premieres livraisons. - Prix de chaque livralson de plancbes.

20

Ouvrage publiè sous les auspices du minislère de l'Instruction publique.

DICTIONNAIRE GENERAL, RAISONNE ET HISTORIQUE DES EAUX ET FORETS, contenant l'analyse des lois, ordonnances, arrêts et instructions, la police et la conservation des forèts; les diversesecit nodes de culture, d'aménagement et d'exploitation; l'architecture navale, la botanique, la minéralogie, etc., appliquées à l'économie forestière, par' M. Baudrillart, chef de diviion a l'administration des foréts. 2 forts volumes in-1. (Ouvrage épuisé el très-rare.)

$70 \mathrm{fr}$.

OSTÉOGRAPHIE, ou description iconographique comparée du squelette et du systeme dentaire des cinq classes d'animaux ver-
tébrés récents et fossiles, pour servir de base a la tèbrés récents et fossiles, pour servir de base à la zoologie et à la stitut (Académie des sciences), professeur d'anatomie comparée au muséum d'histoire naturelle; ouvrage accompagné de planches lithographiées sous sa direction, par MM. J. D. Werner et Delahaye, peintres du Muséum d'histoire naturelle de Paris. $700 \mathrm{fr}$.

Cet ouvrage est publié en 26 fascicules. Chaque fascicule, complet en lui-même, est cntièrement consacré aे un graud genre linnéen, tant sous le rapport iconogrsphiquo que sous le rapport littéraire. Le texte, format in-4, sur papier grand raisin vélin, est livré broché. Les planches, format demi-jesus vélin superîn satiné, sont toutes dessiune étiquette annonģant le contenu de chaque fascicule.

(Onvrage eatièroment termine.)

Vient de paraitre : Fascicule et DFRYrer.

Ce dernier fascicule contient : $1^{\circ}$ les titres et tables du texte de 1'atlas; $9^{\circ}$ une notice sur la vie et les traraux de $\mathrm{M}$. de Blainville; $3^{\circ}$ le mómoire sur 1c cheral, manuscrit inédit de M. de Blainville; $4^{\circ}$ une table générale alphabétique des matières avec renvol au texte et aux plaaches.

OSTEOORAPHIE DES CETAGES, vivants et fossiles; description iconographique du squelette et du systène dentaire de ces animaux ainsi que des documents relatifs à leur histoire naturelle, par MM. Van Beneden, professeur à l'Université de Louvain, et Paul Gervais, membre de l'Institut (Académie des sciences), professeur d'anatomie comparée au Muséum de Paris.

Cette publleation paraitra on 17 lirraisons environ, renfermant chacnne 5 feullles in-4 de texte et 4 planches grand in-follo lithographiées.

En vente : les douze premières livraisons, - Prix de chaque livraison.

$15 \mathrm{fr}$

Destinés à vivre au scin des mers, les Cétacés ont nne certaine ressemblance avec cs poissons: mais ils sont bien éloignés d'avoir la structure anatomique de ces animaux. Leurs poumons conformés comme ceux des mammiferes, l'étévation de leur temperature, leur coeur divisé cn quatre cavités, le volume et la disposition de leur ccrveau, l'Incontestable iatelligence dont ils sont doués, leur mode de reproduction à la fols vivipare etplacentaire : tout, dans leur organisation comme dans leurs fonctions, démontre que ce sont bien des mammiferes, et la présence de mamelles, destinées à ract chent aux vertébrés de cette classe.

Les conditlons spéciales de l'habitat des Cétacés expliquent assez lea particularités qui distinguent ces animaux des autres mammiferes et en font un groupe is la fois natroel el facile à reconnaître; anssi lour étnde présente-t-elle un iaterêt véritable. La chasse ct la pêcho tendant chaque jonr à falre disparaitre ce groupe interessant de mammiferes, il est à supposer qne, dans nn temps rapproche, ils cesseront complètement d'exister. 11 étalt donc urgent d'eu reproduire exactement les caracteres prin-

Un dessinateur spéclal a été envoyé dans les prlncipaux muáes d'Europe pour représeater, d'après nature, toates les pièces existantes sur ce groupe de mammiferes, afin de les coordonner et réunir en uu corps d'ourrage complet.

ZOOLOGIE ET PALEONTOLOGIE FRANÇAISES. Nouvelles recherches sur les animaux vertébrès dont on trouve les ossements enfouis dans le sol de la France, et sur leur comparaison avec les espéces propres aux autres régions du globe, par M. Paul Gervais, membre de l'Institut. $2^{\circ}$ édition. 1 fort volume in $4^{\circ}$ de 600 pages, papier grand raisin, renfermant plusieurs figures dans le texte et accompagnées d'un atlas de 84 planches lithographièes. $100 \mathrm{fr}$.

ZOOLOGIE ET PALÉONTOLOGIE GENERALES, ou nouvelles recherches sur les animaux vertẻbrés vivants et fossiles et comprenant
des documents et mémoires d'anatomie et de paléontologie sur différents groupes, par M. Paul Gervais, membre de l'lnstitut, professeur au Muséum de Paris.

Cette pnblication se composera de 3 volumes format grand in-4. Chaque volume contiendra 500 pages environ avec de nombreuses figures dans le texte et sera accom. pagné d'un atlas de 50 planches litbographiées.

Première série. - Recherches sur l'ancicnneté de l'homme et la période quaternaire. Recherches sur différents groupes de mammiferes, particulièrement sur les ternaire. Recherches sur différents groupes de mammiferes, particulierement sur les animaux quí ont euses de vertébrés ovipares, les uns actuellement existants, les antres éteints, et sur les faunes auxquelles iis appartiennent.

$65 \mathrm{fr}$

Seeonde sèrie. - Remarques an snjet d'un des derniers naturels du Diémen. Description de l'Orsopithecus Bamboliz suivie de remarqnes sur lcs mammireres fossiles de iltalie. Des animaux dont les ossements accompag nent les depôts de chanx phosphatce. Recherches sur l' Euplere, carnirore particulier a la faune de Madagascar dont on avait d'abord fait un genre de l'ardre des Insectivores. Description osteologue de r'Ureus melanoleucus, découvert an Tibct par l'abbé David. Révision des Rhinacéros fossiles, des cberrotins fossiles et genres voisins. Nonveaux détails au sujet des Ėdentés fossiies, ete, etc.

En vente : les trois premières livraisons. - Prix de chaque livraison.

s fr.

JOURNAL DE ZOOLOGIE, renfermant des mémoires originaux sur les différentes branches de l'histoire des animaux vivants et fossiles: mours, distributions géographique et paléontologique, ostéographie, anatomie et physiologie comparées, embriogènie, histologie, tẻratologie, zootechnie, etc., etc., par M. Paul Gervais, membre de l'Institut.

Première série. - Années 1872 à 1877,6 forts volumes grand in-8, accompapés de 120 planches lithogrsphićes et de figures dans le texte. $100 \mathrm{fr}$. Chaque année séparément.

Paris. - Imprimerie Arnous de Rivière, rue Racine, 26. 